\title{
Un centre-ville rose
}

\section{La création d'espaces queerà Hillbrow (Johannesbourg) au temps de l'apartheid}

\author{
Jonathan Botes
}

Traduit de l'anglais par Gilles Baro, Sophie Dulucq et Camille Evrard

\author{
Citer cet article : Botes Jonathan (2021), « Un centre-ville rose. La création d'espaces queer à Hillbrow \\ (Johannesbourg) au temps de l'apartheid », Revue d'Histoire Contemporaine de l'Afrique, n 2, 117-134, en \\ ligne. URL : https://oap.unige.ch/journals/rhca/article/view/02botes \\ Mise en ligne : $1^{\text {er }}$ octobre 2021. \\ DOI : https://doi.org/10.51185/journals/rhca.2021.e556
}

\section{Résumé}

Johannesbourg, plus grande ville d'Afrique du Sud et cœur économique du pays, possède une riche histoire de culture queer. L'article se concentre sur le quartier de Hillbrow, dans les années 1970 et 1980, à une époque où il est considéré comme le centre gay de Johannesbourg. II a pour objectif de montrer comment les homosexuels ont façonné, dans ce quartier du centre-ville, une culture urbaine propre, avec la création de clubs et de bars gays ou gay-friendly, avec la multiplication des fêtes privées et la constitution d'espaces publics de "drague » (cruising). Ces processus sont examinés dans le contexte de l'/mmorality Amendment Act de 1969 qui criminalise les homosexuels. L'article analyse également la dynamique raciale entre homosexuels noirs et blancs, et la façon dont elle a compliqué la production d'espaces gays et queer dans la ville. Basée sur des archives et des entretiens oraux, cette recherche met en lumière l'importance de Hillbrow en tant qu'« espace sûr » (safe space) pour les personnes queer durant les années d'apartheid.

Mots-clés : Johannesbourg ; culture urbaine ; culture queer; gay ; lesbiennes ; drague 
Dès le début des années 1950, le régime d'apartheid affermit son contrôle sur les grandes villes d'Afrique du Sud, principalement par l'imposition d'une ségrégation raciale plus stricte. Johannesbourg, centre économique et ville la plus peuplée du pays, est sans doute la principale zone d'expérimentation de cette politique. Les Noirs sont relégués à la périphérie de la ville afin que le centre-ville soit conforme à l'idéologie de l'apartheid, qui vise à garantir le privilège et le pouvoir aux Blancs. À l'apogée du régime - durant sa « décennie d'or », du début des années 1960 au début des années 1970 -, il semble que ces objectifs soient atteints. Le mouvement anti-apartheid qui a fleuri dans les quartiers noirs de Johannesbourg dans les années 1940 et 1950 est alors réprimé et réduit au silence. Cependant, sous ce vernis de tranquillité et d'ordre urbain, d'autres formes de dissension se cristallisent et, ce faisant, remodèlent les cultures urbaines.

Cet article examine le développement d'une culture queer ${ }^{1}$ à Hillbrow, quartier central de Johannesbourg, et la façon dont celle-ci a contribué à la production d'espaces de transgression remettant en question l'idéal des villes blanches de l'apartheid. Ce régime, basé sur le contrôle, la séparation, l'oppression et la violation des corps noirs², idéalise parallèlement la « blanchité », dont la figure centrale est l'homme blanc hétérosexuel - figure qui légitime les fondements idéologiques racistes et hétéronormatifs du National Party (NP). Cependant, le projet de création d'une communauté blanche culturellement et idéologiquement monolithique, fondée sur les valeurs du nationalisme chrétien, est remise en question sur plusieurs fronts, notamment avec l'émergence d'espaces queer de plus en plus visibles. La culture queerflorissante qui émerge à Hillbrow dans les années 1970 et 1980 est dominée par des hommes gays blancs qui, pour la plupart, ne sont pas explicitement opposés à l'apartheid, et dont les revendications portent plutôt sur la question de la protection des gays. La présence de corps noirs homosexuels dans ces espaces n'est d'ailleurs pas toujours bien accueillie par les Blancs mais, à mesure qu'ils se font plus nombreux dans les années 1980, les caractéristiques de cette culture et des espaces queer de Hillbrow commencent à changer de façon substantielle.

Le présent article vise à combler les lacunes liées à l'absence de recherches approfondies sur la création de ces espaces queer à Johannesbourg. Son ambition est d'analyser comment et pourquoi de tels espaces ont été créés. II vise à mettre en évidence l'importance de l'espace queer en tant qu'espace "safe » pour une catégorie d'individus marginalisés qui, jusqu'à récemment, ont été négligés par l'historiographie sud-africaine. L'article repense le centre-ville comme un espace prisé par les homosexuels, en raison de l'anonymat qu'ils y trouvent et qui leur offre une plus grande sécurité que partout ailleurs. Ainsi Hillbrow et les quartiers environnants deviennent un refuge pour la population homosexuelle de Johannesburg.

La culture queer de la ville se développe parce que les groupes concernés sont en quête de solidarité et d'expériences sexuelles avec des individus eux-mêmes queer, à une époque où ce genre de sexualité est jugé illégitime. Les différentes phases d'urbanisation de l'Afrique du

\footnotetext{
${ }^{1}$ Le terme queer sera utilisé tout au long de cet article en référence à de multiples groupes de personnes qui se définissent comme gays, lesbiennes, trans, etc. Lorsque je ferai référence à un groupe en particulier, ou à des individus, j'utiliserai la terminologie spécifique qui les décrit.

2 Carolin Andy (2017), "Apartheid's Immorality Amendment Act and the fiction of heteronormative whiteness », Tydskrif vir Letturkunde, 54(1), p. 111.
} 
Sud - la ruée vers les mines de Johannesbourg à la fin du XIX $X^{e}$ siècle, l'afflux massif de ruraux dans les villes dans les années 1920 et 1930 - ont attiré des individus des individus d'un type nouveau, éloignés de leur famille, capables de pratiquer ce que Mark Gevisser appelle «l'autonomie personnelle » et, pour certains, de contribuer à la création d'une "culture homosexuelle» avec plus de facilité qu'auparavant ${ }^{3}$. La culture homosexuelle, et plus largement la culture queer, peuvent être considérée comme une contre-culture qui se démarque des « groupes sociaux dominants » de la société hétéronormative ${ }^{4}$.

Katie Mooney explique que « les identités humaines ne sont pas déterminées par un seul facteur hégémonique ", mais plutôt par un ensemble "d'identités comprenant la race, l'ethnicité, le genre et la génération » et, peut-on ajouter, l'orientation sexuelle ${ }^{5}$. Au gré des identités collectives, des contre-cultures se développent, réunissant des individus qui se reconnaissent dans leur différence par rapport à la société dominante. Dans les années 1970 et 1980, Hillbrow devient ainsi l'épicentre de la culture queerà Johannesbourg, voire dans tout le pays. Nous verrons comment femmes et hommes homosexuels ont alors pu créer leurs propres espaces de transgression face à une surveillance croissante de l'État. C'est en effet une période où une culture queer urbaine s'épanouit, comme en témoigne l'ouverture d'une multitude de clubs, de bars et de restaurants à Hillbrow et alentour, destinés à cette communauté florissante. Les hommes gays continuent de plus belle à pratiquer le cruising tandis que certains domiciles privés deviennent des lieux de rencontre pour les lesbiennes ${ }^{7}$. En se concentrant sur la production de ces espaces, l'article souhaite examiner le caractère transgressif de la culture queer au sein d'un système politique répressif, raciste et hétéronormatif.

En nous basant sur des archives et des entretiens menés avec des figures clés de la communauté queer de Johannesburg, nous mettrons ici en lumière les interactions quotidiennes des personnes queer à Hillbrow, et la manière dont elles ont pu contourner les lois oppressives et créer des espaces sûrs au sein desquels elles ont pu exprimer leur sexualité. Nous nous appuierons principalement sur les importantes collections du centre Gay and Lesbian Memory in Action (GALA) de l'université du Witwatersrand, centrées sur les multiples aspects de la vie des personnes queer en Afrique du Sud. Nous recourrons aussi à des entretiens oraux, ainsi qu'a des retranscriptions d'entretiens trouvées dans les archives de GALA. Ces collections documentaires sont uniques en Afrique du Sud : elles conservent les archives de militants gays et lesbiens qui ont été, en grande partie, parrainés par GALA, ainsi que des retranscriptions d'entretiens historiques, des articles de revues relatifs à l'histoire des gays et des lesbiennes en Afrique du Sud, des objets personnels tels que des journaux intimes ou encore des correspondances. Ce centre documentaire est essentiel à la compréhension et à la recherche de l'histoire des personnes queer dans le pays.

\footnotetext{
3 Gevisser Mark (1994), «A Different Fight for Freedom: A History of South African Lesbian and Gay Organisation from the 1950s to 1990s ", in M. Gevisser et E. Cameron (dir.), Defiant Desire: Gay and Lesbian Lives in South Africa, Braamfontein, Ravan Press, p. 18.

4 Mooney Katie (2006), "Die Eendstert Euwel" and societal responses to white youth sub-cultural identities on the Witwatersrand, 1930-1964, Thèse de doctorat en histoire, Université de Witwatersrand, p. 15.

5 Mooney K., "Die Eendstert Euwel...", op. cit., p. 19.

${ }^{6}$ Note des traducteurs : terme plus ou moins équivalent du mot français " drague », qui désigne la recherche d'un ou de plusieurs partenaires sexuels. Voir Redoutey Emmanuel (2009), "Drague et cruising. Géométaphores d'un mouvement exploratoire », Echogéo, 5, en ligne. URL : https://journals.openedition.org/echogeo/3663 (consulté le 4 septembre 2021).

7 Gevisser Mark (1994), « A Different Fight for Freedom... », art. cité, pp. 24-25.
} 
La littérature existante sur l'histoire du mouvement et des personnes queer en Afrique du Sud s'attaque aux questions relatives à l'hétéronormativité et à leur existence dans des espaces façonnés par l'hétérosexualité. Une recherche essentielle sur cette culture est sans aucun doute l'ouvrage collectif Defiant Desire: Gay and Lesbian Lives in South Africa de Mark Gevisser et Edwin Cameron, paru à l'aube de la démocratisation sud-africaine. II s'agit d'une publication pionnière qui lève le voile sur la vie des lesbiennes et des gays, toutes races et classes confondues, en documentant les politiques sexuelles ainsi que l'agentivité érotique au temps de l'apartheid. Depuis lors, le corpus d'études portant sur les personnes queer s'est considérablement étoffé, même si une grande partie des travaux a eu tendance à se concentrer sur des questions générales affectant la vie de ces catégories en Afrique du Sud, telles que la discrimination et la violence persistantes à leur encontre. Un autre pan important de la recherche s'est intéressé à l'activité politique des communautés queer, en particulier à leurs implications diverses dans les luttes anti-apartheid et dans les mouvements de l'ère démocratique ${ }^{8}$.

Cet article a quant à lui l'ambition d'analyser la culture queer dans un lieu particulier, qui a reçu peu d'attention dans l'historiographie. Les espaces urbains où les personnes queer se sont regroupées, en particulier durant l'apartheid, constituent un aspect important de cette recherche. Pendant la Seconde Guerre mondiale, Joubert Park devient un quartier réputé pour ses bars, souvent fréquentés par des hommes homosexuels qui espèrent y rencontrer des soldats en permission. Hillbrow et Joubert Park, avec leurs logements bon marché, leur forte densité et leur proximité avec le Central Business District (CBD), émergent peu à peu comme des lieux privilégiés pour les interactions entre homosexuels ${ }^{9}$. Ces quartiers sont également marqués par un afflux croissant d'hommes noirs, ce qui crée des occasions plus régulières d'interactions entre homosexuels blancs et noirs. Cette transformation importante de Hillbrow contribuera d'ailleurs à la fin de la période étudiée ici à l'exode des Blancs, exode qui entraînera à son tour l'abandon progressif des clubs et des bars.

D'une superficie d'un kilomètre carré environ, Hillbrow est historiquement une zone densément peuplée, caractérisée par la présence de nombreux immeubles élevés. Après la suppression des restrictions de hauteur imposées aux bâtiments de Johannesburg dans les années 1950, le quartier connaît une « poussée immobilière » marquée par la prolifération des appartements résidentiels ${ }^{10}$. Entre 1945 et 1965, le nombre d'appartements augmente de $250 \%$. Bien que tous les propriétaires et habitants soient blancs, on trouve également dans le centre-ville des espaces occupés par des Noirs: le Native (Urban Areas) Amendment Bill

\footnotetext{
8 Voir : Nicol Julia (1991), "Organisation of Lesbian and Gay Activists », Agenda: Empowering Women for Gender Equity, 7(11), pp. 45-46 ; Gevisser Mark et Cameron Edwin (1994), « Defiant Desire », in M. Gevisser et E. Cameron (dir.), Defiant Desire: Gay and Lesbian Lives in South Africa, Braamfontein, Ravan Press, pp. 3-13; Morrell Robert (1998), « Of Boys and Men: Masculinity and Gender in Southern African Studies», Journal of Southern African Studies, 24(4), pp. 605-630; Reddy Vasu (1998), « Negotiating Gay Masculinities », Agenda: Empowering Women for Gender Equity, 37, pp. 65-70 ; Cock Jacklyn (2003), " Engendering Gay and Lesbian Rights: The Equality Clause in the South African Constitution », Women's Studies International Forum, 26(1), pp. 35-45; Hoad Neville, Karen Martin et Graeme Reid (dir.) (2005), Sex and Politics in South Africa, Cape Town, Double Storey Books ; Carolin A., « Apartheid's Immorality Act... », art. cité ; Conway Daniel (2009), "Queering Apartheid: The National Party's 1987 'Gay Rights' Election Campaign in Hillbrow », Journal of Southern African Studies, 35(4), pp. 849-863; du Pisani Kobus (2012), "Shifting Sexual Morality? Changing Views on Homosexuality in Afrikaner Society during the 1960s », Historia, 57(2), pp. 182-221.

${ }^{9}$ Gevisser M., « A Different Fight for Freedom... », art. cité., p. 19.

10 Morris Alan (1999), "Race Relations and Racism in a Racially Diverse Inner City Neighbourhood: A Case Study of Hillbrow, Johannesburg », Journal of Southern African Studies, 25(4), pp. 670-671.
} 
$\left(n^{\circ} 12\right)^{11}$ de 1954 autorise en effet l'installation d'un nombre maximum de "cinq Noirs au sommet d'un bloc d'appartements ou de tout autre bâtiment, dans les zones urbaines ${ }^{12}{ }^{2}$. Ces «logements dans le ciel» sont principalement réservés aux travailleurs domestiques, instaurant au cœur de la plupart des relations interraciales de Hillbrow une dynamique entre « maître » et « serviteur ».

En raison du grand nombre d'appartements relativement bon marché et de leur proximité avec le CBD, de nombreux résidents de Hillbrow sont jeunes, célibataires, ou bien sont de jeunes couples mariés ${ }^{13}$. La forte densité du quartier et la prédominance de jeunes gens majoritairement célibataires contribuent au dynamisme urbain et à l'exubérance de la vie nocturne. Historiquement, le centre-ville a toujours été caractérisé par une attitude permissive vis-à-vis des activités peu conformes à l'idéologie conservatrice du National Party, y compris à l'égard des sexualités non hétéronormées. L'arrivée d'une jeune génération après la Seconde Guerre mondiale génère un appétit grandissant pour des clubs et des bars échappant aux cadres définis par le NP. Ayant récemment quitté la maison familiale, cette nouvelle génération s'immerge rapidement dans les fêtes bohèmes de Hillbrow.

Nous souhaitons mettre en lumière le développement des espaces queer dans le quartier en proposant une périodisation. Nous montrerons comment ils ont évolué, à mesure que les lieux informels se sont transformés en lieux plus structurés. Le déplacement des personnes queer des lieux de drague publics vers des clubs organisés marque le début de constitution d'une communauté visible et constitue un point de départ idéal pour l'examen des espaces queer de la ville. Les barrières raciales existant sous le régime de l'apartheid ont joué un rôle crucial dans la structuration de ces espaces, et l'échec des politiques raciales dans les années 1980 constitue un autre point focal dans la progression des espaces queer à Johannesburg. Bien qu'il ait existé dans le centre-ville des lieux considérés comme ouverts aux queer, ces derniers ont toujours dû naviguer dans ces espaces de manière à assurer leur sécurité. L'analyse portera donc sur la façon dont ces personnes ont adapté leurs vêtements pour circuler en ville et pour exprimer leurs propres intérêts sexuels sans attirer l'attention d'une société hétéronormative.

\section{Cruising et clubs}

Les lieux de cruising ont toujours été importants pour les interactions sexuelles entre hommes homosexuels. Dans les années 1940 et 1950, à Johannesbourg, ces espaces deviennent populaires en raison de la présence massive de soldats dans le centre-ville et ses environs immédiats ${ }^{14}$. Les parcs publics, les toilettes de Park Station, le bureau de poste de Joubert Park et Union Grounds ${ }^{15}$ sont quelques-uns des lieux bien connus où les hommes peuvent trouver

\footnotetext{
${ }^{11}$ Cette loi est l'une des principales lois de l'apartheid, instauré le 27 avril 1950. Elle oblige différents types de populations à résider dans des zones urbaines d'habitation prédéfinies.

12 Mather C. (1987), "Residential Segregation and Johannesburg's "Locations in the Sky" », South African Geographical Journal, 69(2), 
d'autres hommes prêts à explorer, rapidement et discrètement, leur sexualité, parfois par-delà les frontières raciales ${ }^{16}$.

Hillbrow et le quartier adjacent de Joubert Park sont des destinations prisées par une jeune génération d'homosexuels, en raison de leurs logements peu coûteux, de leur proximité avec le $C B D$ et de leur réputation d'espaces de transgression. Pendant la Seconde Guerre mondiale, leurs bars sont fréquentés par des homosexuels qui espèrent rencontrer des soldats en permission stationnés à proximité ${ }^{17}$. Percy ${ }^{18}$, un informateur gay noir, se souvient de l'importance de ces soldats de passage lors de l'exploration de sa propre sexualité. Dans les années 1940 et 1950, Percy, ainsi que d'autres hommes noirs des townships de la périphérie de Johannesburg, se rendent à Union Grounds pour avoir des relations sexuelles avec des soldats. En longeant la clôture d'Union Grounds lorsque la nuit est trop sombre pour discerner ce qui s'y passe, Percy et les autres sont abordés par des soldats en patrouille, qui leur demandent: "C'est combien ? ». La réponse est toujours "vingt-six » (" deux shillings et six pence »). Les soldats blancs s'acquittent alors de la somme et les couples se livrent à des rapports sexuels avec pénétration à travers le "grillage à mailles en losange ${ }^{19}$ ». Bien qu'il s'agisse d'une transaction, Edwin Cameron - un homosexuel blanc qui a participé à la création de la première Gay Pride en Afrique du Sud - estime que ces hommes ne se considèrent pas comme des travailleurs du sexe, mais voient plutôt leurs interactions comme l'un des rares moyens d'avoir des relations sexuelles masculines discrètes par-delà les frontières raciales ${ }^{20}$. Paola Tabet confirme cette interprétation de Cameron en notant que les normes culturelles diffèrent et que, dans certaines cultures, le fait de recevoir de l'argent et des gains matériels en échange de rapports sexuels n'est pas toujours considéré comme du travail sexuel ${ }^{21}$.

Percy rappelle aussi l'importance du bureau de poste de Joubert Park, un autre lieu de cruising populaire dans le secteur de Hillbrow, comme un « lieu de rencontre » qui le met en contact avec des hommes blancs capables de lui offrir un refuge vis-à-vis de la police :

Même à Joubert Park, la police faisait des descentes et arrêtait beaucoup de garçons qui se déguisaient au bureau de poste. C'était le lieu de rencontre. Parce que vous y alliez tard dans la soirée et les Blancs venaient chercher les gens là-bas. J'allais avec les garçons [noirs] [à la poste] mais je n'étais pas intéressé par... les Blancs. Je voulais juste y aller parce que ces garçons [noirs] voulaient que nous y allions en groupe... Puis j'ai rencontré un certain nombre de Blancs... qui récupéraient des garçons à la gare et les invitaient chez eux en disant : «Viens Percy, nous avons tellement de garçons, tu peux choisir $\gg .22$

Les homosexuels encore "dans le placard ${ }^{23}$ ", qui ne souhaitent pas se fondre dans la culture gay de Johannesburg, trouvent quant à eux une forme d'anonymat dans ces espaces.

\footnotetext{
${ }^{16}$ Gay and Lesbian Memory in Action (GALA), AM3160(A), B2.2.3, Queer Tour Drafts/notes/routes.

17 Gevisser M., «A Different Fight for Freedom... », art. cité., p. 19.

18 Pseudonyme.

19 Percy. Interview menée par Jonathan Botes, Johannesbourg, 30 juillet 2019, transcription en possession de l'auteur.

20 E. Cameron. Interview menée par Jonathan Botes, Johannesbourg, 2 juillet 2019, transcription en possession de I'auteur.

21 Tabet Paola (2012), "Through the looking glass: sexual-economic exchange », in F. Grande Omokaro et F. Reysoo (dir.), Chic, chèque, choc : transactions autour des corps et stratégies amoureuses contemporaines, Genève, Graduate Institute Publications, p. 40.

22 Percy. Interview menée par Jonathan Botes, Johannesbourg, 30 juillet 2019, transcription en possession de l'auteur.

23 Personnes queer n'ayant pas parlé de leur sexualité à autrui, généralement à leurs proches.
} 
En parlant de sa propre expérience du cruising dans les années 1980, Levi ${ }^{24}$, un homosexuel blanc de la banlieue, se souvient du secret qui était recherché lorsque l'on draguait :

Je veux dire qu'il n'y avait pas 42 caméras à chaque coin de rue à l'époque... il y avait une sorte de monde souterrain subtil... C'est un peu bizarre, mais les toilettes publiques étaient plutôt disponibles à Hillbrow à l'époque... et vous preniez le risque de rencontrer des gens là-bas. ${ }^{25}$

En dépit des risques encourus, ces rencontres sexuelles se poursuivent tout au long des années d'apartheid. Et les dangers sont alors nombreux. Les lieux de cruising deviennent des espaces de violence à l'égard des homosexuels, tandis que des histoires circulent sur la façon dont certains hommes y sont "agressés ou battus ${ }^{26}$ ». Les dangers du cruising sont souvent ignorés par les intéressés, au regard de la garantie d'accès à des expériences sexuelles qu'offre cette pratique à tous ceux qui sont en quête de gratification instantanée.

À la suite de la promulgation de l'amendement sur la Loi sur l'Immoralité (Immorality Amendment $A c t$ ) de $1969^{27}$, le cruising devient particulièrement risqué, la police bénéficiant de pouvoirs de surveillance accrus pour réprimer I'homosexualité. La loi vise les homosexuels qui se livrent à toute activité sexuelle en présence de deux personnes ou plus ${ }^{28}$. Ses conséquences immédiates, tout au long des années 1970, consistent en une surveillance et un contrôle plus stricts des zones urbaines gays : lieux de cruising, clubs, bars, fêtes, etc. ${ }^{29}$. Les embuscades de la police deviennent monnaie courante ${ }^{30}$. La police justifie ces «mesures répressives à l'encontre des lesbiennes, des gays et des bisexuels [blancs] par la volonté de traiter l'activité homosexuelle dans les lieux publics comme une nuisance et d'accuser les transgresseurs de délits sexuels », sans lien particulier avec la sexualité elle-même, mais plutôt en invoquant des motifs d'indécence publique, d'agression sexuelle et de racolage immoral ${ }^{31}$.

La revendication d'un espace sûr pour les personnes queernaît de ces expériences vécues et de la volonté de reconnaissance exprimée par certains gays et lesbiennes, qui se sentent aliénés dans la ville. Mais le "droit à la ville ne signifie pas tous les droits pour tous » et, en tant que tels, les corps noirs queer sont exclus de ces espaces au cours des années 1970, alors même que les hommes gays blancs se réservent l'accès exclusif à leurs propres espaces ${ }^{32}$. La revendication d'un "droit à la ville » prend d'abord forme dans les clubs de Hillbrow, qui commencent à prospérer au début des années 1970. Kanika Batra suggère que cette revendication est alors devenue possible pour deux raisons : la première est que Hillbrow est connu pour son atmosphère bohème, née de l'urbanisation croissante du centre-ville par de

\footnotetext{
24 Pseudonyme.

25 Levi. Interview menée par Jonathan Botes, Johannesbourg, 19 août 2019, transcription en possession de l'auteur.

26 Ibid.

27 À l'origine, I'/mmorality Act est constitué de deux lois. La première, en 1927, interdit les rapports sexuels entre Blancs et Noirs, et est amendée en 1950 pour interdire toute relation entre Blancs, Noirs et Coloured. La deuxième loi de 1957 confirme à nouveau cette interdiction et interdit le proxénétisme, les maisons de prostitution et les rapports sexuels avec des personnes de moins de 16 ans. L'amendement de 1969 introduit de nouveaux délits, notamment les rapports sexuels entre un homme et un garçon de moins de 19 ans, ainsi que les rapports entre hommes au cours de « fêtes » - soit tous les rassemblements de plus de deux personnes.

28 Gevisser M., «A Different Fight for Freedom... », art. cité., p. 35

29 Retief Glen (1994), "Keeping Sodom out of the Laager: The Policing of Sexual Minorities in South Africa », in M. Gevisser et E. Cameron (dir.), Defiant Desire: Gay and Lesbian Lives in South Africa, Braamfontein, Ravan Press, p. 103.

30 Republic of South Africa, Annual Report of Criminal Offences, Pretoria, Central Statistical Services, 1982.

31 Retief Glen (1994), « Keeping Sodom out of the Laager... », art. cité, p. 106.

32 Marcuse Peter (2016), "Whose Right(s) to what City? », in N. Brenner, P. Marcuse et M. Mayer (dir.), Cities for People, Not for Profit: Critical Urban Theory and the Right to the City, Oxon, Routledge, p. 35.
} 
jeunes Blancs progressistes ${ }^{33}$; la seconde raison peut être attribuée à une relative tolérance de la police à l'égard des clubs, afin de pouvoir contrôler plus facilement les activités homosexuelles et de les tenir à l'écart du grand public. Cela a pour effet d'éloigner les homosexuels des lieux de cruising et de les faire rentrer dans les clubs:

Le développement d'une vie commerciale gay, sous la forme de clubs et de bars s'est accompagné, dans le cas de Hillbrow, à Johannesburg du moins, de l'officialisation d'une zone où les homosexuels avaient toujours vécu dans un "quartier gay » clairement identifiable... et [bénéficiaient] d'un niveau de tolérance inédit de la part des autres habitants. Au fur et à mesure que le quartier s'est développé, les autorités ont décidé de considérer la zone densément peuplée de Hillbrow comme un "lieu de rencontre pour les homosexuels »... en choisissant de ne pas exercer la même pression dans ses rues que sur d'autres espaces publics. ${ }^{34}$

Batra affirme que le National Party et la police trouvent plus aisé de contrôler les mouvements des homosexuels s'ils restent à l'intérieur, cachés du reste de la société ${ }^{35}$. Hannah, qui dirige un club pour homosexuels à Johannesburg au début des années 1970, se souvient d'un officier de la brigade des mœurs qui venait régulièrement la surveiller, ainsi que son club : « II passait de temps en temps pour prendre sa bouteille de whisky. II disait qu'il n'avait pas d'objection vis-à-vis du club, parce qu'il préférait savoir que tout le monde se trouvait sous le même toit plutôt qu'à Zoo Lake ou à Joubert Park ${ }^{36}$ ». Bien que la police ait connaissance de l'existence de ces clubs, elle les autorise à poursuivre leurs activités, car il semble que l'intention est « de mettre les [homosexuels] à l'écart de la société... tant que les [homosexuels] gardaient [leurs] sales habitudes en dehors de la rue, ils étaient à l'abri » de poursuites ${ }^{37}$. Cette idée de les maintenir "sous le même toit » souligne l'état d'esprit de la police, qui pensait qu'il était plus facile de contrôler la population queer de Johannesburg si elle était regroupée dans un même lieu plutôt que dispersée dans toute la ville.

En raison du caractère bohème de Hillbrow et de l'afflux de Blancs progressistes en ville, la culture homosexuelle se développe relativement librement. Les clubs, bars et restaurants gays qui prennent de l'importance au cours des années 1970 réussissent à se maintenir en tant $q u^{\prime}$ ' entreprises commerciales exclusivement gays » et offrent un espace où une clientèle gay peut se rassembler ${ }^{38}$. La communauté gay de Hillbrow, en pleine expansion, contribue à l'ouverture de plusieurs de ces clubs populaires au fil de la décennie : le Dungeon ouvre ses portes quelques semaines après l'adoption de I'Immorality Amendment Act de 1969; le Rocky's au Continental et le New Library sont ouverts au début des années 1970 ; plus tard, le Butterfly à I'hôtel Skyline devient l'un des lieux gays les plus fréquentés de la ville. Quant au Together Bar, ou T-Bar comme on l'appelle souvent, il accueille à partir de 1974 une clientèle de lesbiennes à l'hôtel Hilton Court de Hillbrow ${ }^{39}$.

\footnotetext{
${ }^{33}$ Batra Kanika (2016), « Worlding Sexualities under Apartheid: From Gay Liberation to a Queer Afropolitanism », Postcolonial Studies, 19(1), p. 40

34 Ibid, p. 38.

35 Ibid, p. 37.

36 Ibid.

37 Gevisser M. et Cameron E, «Defiant Desire ... », art. cité, p. 37.

38 Batra K., « Worlding Sexualities under Apartheid ... », art. cité, p. 25.

39 Ibid., pp. 39-41.
} 
Comme on vient de le voir, c'est en 1969 que Ronnie Oelofsen ouvre le Dungeon Club, I'un des plus anciens clubs gays de Johannesburg. L'établissement commence ses activités quelques semaines après la promulgation de la loi de 1969. Le soir de son ouverture, il diffuse de la musique sakkie-sakkie ${ }^{40}$, ce qui contribue à lui assurer une clientèle majoritairement afrikaner. Oelofsen soutient que le Dungeon a pu fonctionner grâce au respect d'un ensemble de règles strictes : "Tant qu'il n'y avait pas de mineurs dans les locaux et que vous ne vendiez pas d'alcool, vous étiez en sécurité. La seule fois où j'ai fait l'objet d'une descente de police, $c^{\prime}$ est lorsque, un dimanche soir, j'ai projeté un film interdit $»^{41}$.

Il est possible qu'Oelofsen ait pu maintenir le club en activité sans trop de questions de la part de la police grâce à ses relations avec les conseillers municipaux locaux, qu'il autorise à utiliser l'établissement pour organiser des fêtes à destination des personnes âgées ${ }^{42}$. Quant au Butterfly, situé dans I'hôtel Skyline sur Pretoria Street, il devient rapidement l'un des clubs gays les plus fréquentés de Hillbrow, bien connu pour être un lieu de rencontre bon marché où l'on pouvait trouver des hommes en train de faire du cruising après le déclin de Park Station ${ }^{43}$. Tout au long des années 1970, de nombreux bars et clubs gays ouvrent donc leurs portes dans le secteur de Hillbrow, et le quartier acquiert la réputation d'être gay-friendly. Se constituent alors des espaces où les hommes homosexuels ont la possibilité de construire leur propre contreculture, au sein d'un État hétéronormatif modelé en profondeur par l'apartheid ${ }^{44}$.

\section{Du rose au gris}

Alors que de nombreux homosexuels blancs peuvent facilement accéder aux clubs et aux bars de Hillbrow dans les années 1970, les hommes noirs et pauvres n'ont pas la possibilité d'y pénétrer et sont contraints à faire du cruising, pratique qui se maintient dans la durée ${ }^{45}$. La stratification sociale dans les clubs et aux bars réservés aux Blancs, et dont l'entrée est payante, contribue à ce que la majorité des personnes présentes y soient des homosexuels blancs de la classe moyenne. Robert Morrell note que «les hommes de la classe ouvrière, les Noirs et les homosexuels ont été exclus des [...] masculinités hégémoniques », mais qu'ils se sont retrouvés à divers moments protégés par le «parapluie de la masculinité hégémonique »: ils ont alors contribué à exclure d'autres acteurs sociaux, notamment les femmes, car ils bénéficiaient des structures patriarcales de la société ${ }^{46}$. Morrell souligne que la masculinité hégémonique produite par l'État est constamment contestée, ce qui est une évidence dans les clubs gays de Hillbrow des années $1970^{47}$. Mais alors que les homosexuels blancs défient l'hétéronormativité de l'État en revendiquant un espace à Hillbrow, ils n'en reproduisent pas moins de la discrimination raciale et sexuelle: si les Blancs pauvres ont accès à des bars moins chers à Hillbrow, cela est refusé aux homosexuels noirs. Au moins jusqu'au début des années 1980, ces

\footnotetext{
40 Musique de danse folklorique traditionnelle afrikaans.

41 Gevisser M., « A Different Fight for Freedom... », art. cité., p. 37.

42 Ibid., p. 40.

43 Ibid., pp. 40-41.

44 Carolin A., « Apartheid's Immorality Act ... », art. cité, p. 114.

45 Ibid., p. 37.

46 Morrell R., « Of Boys and Men ... », art. cité, p. 608.

47 Ibid., p. 609.
} 
espaces maintiennent donc la ségrégation de l'apartheid, reflétant la politique de la plupart des Blancs de l'époque - y compris parmi les homosexuels.

La réputation du quartier comme «zone de divertissement de la banlieue blanche » commence à se dégrader au début des années 1980, alors que le régime d'apartheid est attaqué sur plusieurs fronts ${ }^{48}$. La lutte des townships contre l'apartheid atteignant de nouveaux sommets, l'État est de moins en moins capable de faire appliquer les éléments centraux de la législation de l'apartheid, tels que le Group Areas Act (Loi sur les zones réservées) ou le contrôle des flux de population. L'aggravation de la pénurie de logements dans les zones noires et la volonté des propriétaires de louer leurs appartements à des locataires noirs poussent un nombre croissant de ces derniers à rechercher un logement à Hillbrow ${ }^{49}$. Leur afflux dans le centre-ville ainsi que la fuite des habitants blancs transforment la ville cosmopolite, autrefois blanche, en une zone " grise » de Johannesburg: ni blanche ni noire, mais multiraciale.

Edwin Cameron se souvient de Hillbrow dans les années 1980 comme d'un « carrefour de classes et de races ", avec des hommes «métis plutôt qu'africains » fréquentant désormais le quartier ${ }^{50}$. C'est ce que suggèrent les recherches, mais Levi s'en souvient différemment, affirmant qu'il n'a jamais vu beaucoup de non-Blancs à Hillbrow à cette époque ${ }^{51}$. Saul Dubow évoque pour sa part ce passage au «gris »:

Dans le centre-ville de Johannesburg, le quartier cosmopolite de Hillbrow a commencé à devenir "gris " au cours des années 1980, car les Indiens et les Métis (coloured) à la recherche d'un appartement, suivis par les Africains, ont ignoré les dispositions strictes de la loi Group Areas Act... Dans la géographie mouvante des espaces ségrégués, le gouvernement abandonnait effectivement le contrôle du centre-ville de Johannesburg, sachant que sa véritable base de soutien urbain était essentiellement banlieusarde. ${ }^{52}$

L'intégration raciale progressive du quartier suscite l'inquiétude de nombreux hommes politiques de droite, mais elle est saluée par les progressistes, tandis qu'elle fait naître des opinions contrastées parmi les Sud-Africains blancs. La crise économique et politique des années 1970, ainsi que les divergences de vue sur la mise en œuvre de la politique d'apartheid, entraînent une division au sein du National Party entre les verligtes ${ }^{53}$, plus pragmatiques et réformistes, et les verkramptes ${ }^{54}$, plus rigides et conservateurs. L'ascension des verligtes au début des années 1980 contribue au déclin des poursuites pour violation du Group Areas Act, avec seulement trois personnes poursuivies dans le centre-ville de Johannesburg en 1981. Les verligtes réalisent que la survie et la croissance du National Party dépendent en fin de compte de l'abandon des principes fondamentaux de l'apartheid, ainsi que de «la nécessité pour le gouvernement d'élargir sa base de soutien potentielle » en augmentant l'accès des Indiens et des Métis au Parlement ${ }^{55}$. À cette fin, en 1984, le parlement tricaméral intègre les Indiens et les

\footnotetext{
48 Morris Alan (1999), Bleakness and Light: Inner-City Transition in Hillbrow, Johannesburg, South Africa, Witwatersrand University Press, p. 82.

49 Morris A., « Race Relations ... », art. cité, p. 673.

50 E. Cameron. Interview menée par Jonathan Botes, Johannesbourg, 2 juillet 2019, transcription en possession de l'auteur.

51 Levi. Interview menée par Jonathan Botes, Johannesbourg, 19 août 2019, transcription en possession de l'auteur.

52 Dubow Saul (2014), Apartheid, 1948-1994, United Kingdom, Oxford University Press, p. 242.

53 Traduit approximativement par les « éveillés ».

54 Traduit approximativement par « conservateurs».

55 Morris A., « Race Relations ... », art. cité, p. 673.
} 
Noirs dans «le parlement central, par le biais de chambres séparées ${ }^{56}$ ». Ces réformes superficielles, qui entérinent l'impossibilité de maintenir l'apartheid, ne bénéficient que d'un soutien minimal parmi les Blancs, qui ont alors tendance à se replier sur eux-mêmes dans des enclaves sécurisées entièrement blanches.

Des rumeurs faisant état d'une hausse de la délinquance et de criminalité, de la baisse des prix de l'immobilier et de la crainte du mélange racial dans le centre-ville conduisent à une «fuite des Blancs»: de nombreux habitants blancs du centre-ville s'installent dans les banlieues huppées du nord de Johannesburg. La «transformation raciale » de Hillbrow est rapidement associée à la dépréciation et au déclin du quartier tout au long des années $1980^{57}$. L'exode des Blancs est inextricablement lié à la fuite des capitaux, qui a vu « de grandes entreprises commerciales, des entreprises minières, des banques et autres institutions financières, ainsi que des sociétés immobilières » quitter le centre-ville pour s'installer dans les banlieues nord en voie d'urbanisation rapide ${ }^{58}$. Cette migration de la classe moyenne blanche, y compris de la communauté blanche homosexuelle, provoque l'abandon d' « anciens bars et boîtes de nuit emblématiques de Hillbrow [...], comme le Skyline ${ }^{59}$ ». La communauté queer de la classe moyenne blanche se disperse dans la banlieue nord, privant le quartier de l'identité queer, florissante sur le plan commercial, qui l'avait nourri tout au long des années 1970.

Jusqu'au début de la décennie 1980, les Noirs queer appréhendaient généralement de pénétrer dans cet espace queer réputé. Originaire de Soweto, Paul Mokgethi se souvient avoir éprouvé de la peur les premières fois qu'il s'est rendu au Butterfly, un bar gay de Hillbrow:

[I] y avait tous ces hommes blancs qui venaient là. Alors nous avions peur d'y aller, nous avions peur d'entrer dans cet endroit. On se pressait les uns les autres, on allait voir ce qui se passait. Je ne veux pas y aller, j'ai peur. ${ }^{60}$

S'Bu se souvient avoir été harcelé par un videur parce qu'il embrassait publiquement sa petite amie, confirmant ainsi ses craintes de ne pas être en sécurité au centre-ville. L'incident se produit avant la transition de $S^{\prime} B u$, de sorte que sa compagne et lui sont perçus comme deux filles noires s'embrassant dans un espace considéré comme ouvertement gay. Pourtant, le videur cherche à les intimider et leur intime l'ordre de ne pas "faire cette merde ici61 ». Les changements politiques du pays se manifestent également dans l'espace blanc « formel » de Johannesburg. Paul et ses amis tous Noirs finissent par exemple par avoir accès au Butterfly et se lient d'amitié avec le barman, en raison de leur orientation sexuelle. Ils deviennent des habitués, mais sont tenus à l'écart de la clientèle blanche. L'espace du Butterfly, comme celui de nombreux de bars et clubs gays de Hillbrow, est ainsi segmenté en différents groupes : dans un coin, on trouve des lesbiennes (blanches) butch et des lesbiennes (blanches) femmes ${ }^{62}$ dans

\footnotetext{
56 Ibid.

57 "Rather share the franchise than the pool », Frontline, 30 avril 1989.

58 Murray Martin J. (2008), Taming the Disorderly City: The Spatial Landscape of Johannesburg after Apartheid, Ithaca and London, Cornell University Press, p. 127.

59 Banham Hugo (2017), "Mapping the Black Queer Geography of Johannesburg's Lesbian Women through Narrative », PINS (Psychology in Society), 55, en ligne. URL: http://www.scielo.org.za/scielo.php?script=sci_arttext\&pid=S1015-60462017000300006 (consulté le 8 août 2021).

60 GALA, AM3160 (B): C4.1.1, Joburg Tracks, Tracks Interview: Paul.

61 S'Bu Kheswa. Interview menée par Jonathan Botes, Johannesbourg, 4 octobre 2019, transcription en possession de l'auteur.

62 Butch et femme sont des termes utilisés dans la contre-culture lesbienne pour qualifier une apparence et une identité masculine (butch) ou féminine (femme), avec les traits, les comportements, les styles, la perception de soi, etc., qui y sont associés.
} 
un autre ; une autre partie du bar peut accueillir des gays originaires de Doornfontein, tandis que ceux de Melville se regroupent ailleurs; et les gens de la banlieue sud se retrouvent dans un autre recoin de l'établissement. Dans les années 1980, les clients noirs sont partie prenante de cette mixité, en raison de l'échec de la politique raciale qui se constate à Hillbrow ; mais la place qui est attribuée à la stratification sociale dans les bars et des clubs l'est en fonction de la race plutôt que de la classe ou de l'origine ${ }^{63}$.

Les habitants vivent différemment cette évolution du quartier du « rose » vers le " gris », en fonction de leur propre manière de circuler dans l'espace. Lorsqu'on l'interroge sur la présence accrue de corps noirs et de personnes de couleur à Hillbrow, Levi ne se souvient pas de l'avoir remarquée dans les années 1980, probablement en raison des clubs qu'il fréquentait ; mais dans les années 1990, il pense que Hillbrow a déjà commencé à avoir la réputation d'un quartier dangereux, ce qui entraîne la réduction du nombre de clubs et de bars gays qu'il estime désormais pouvoir fréquenter en toute sécurité ${ }^{64}$.

Si les années 1980 marquent ce passage du rose au « gris », les années 1990 sont quant à elles marquées par le déclin puis la disparition d'un centre-ville rose et multiracial. Les habitants queer noirs qui se sont installés à Hillbrow à cette époque se font plus actifs dans les clubs et les bars du quartier. Le Skyline, après avoir décliné à la suite de l'exode des Blancs, redevient le lieu de prédilection de nombreux queer noirs et, dans la seconde moitié des années 1980, ses clients sont à $95 \%$ noirs. L'augmentation du nombre de Noirs à Hillbrow a "fondamentalement modifié la clientèle» des clubs et des bars, qui accueillent majoritairement des personnes queer noires, ainsi que quelques Blancs ${ }^{65}$.

\section{Négocier les risques en ville}

Malgré la croissance des clubs gays, le mode de vie queer continue à être potentiellement dangereux. C'est particulièrement vrai pour les femmes queer, qui choisissent souvent d'organiser des fêtes privées à domicile. Pour y accéder, les participantes doivent être invitées par une connaissance, et ces fêtes s'organisent sur une base professionnelle: enseignantes, infirmières, avocates ou autres ${ }^{66}$. Hannah se souvient que les femmes queer se sentent à l'abri des descentes de police tant qu'il n'y a pas d'hommes présents, car les hommes gays attirent souvent l'attention indésirable des voisins ${ }^{67}$. Si la majorité des activités sociales lesbiennes se déroulent à domicile, il existe quelques espaces publics « sûrs », le plus important d'entre eux étant dirigé par une femme nommée Chick Venter. Situé près de Park Station, club « sauvage » (rough) aux «murs couverts de résilles et de bouteilles » et fréquenté par une clientèle essentiellement masculine, le lieu est l'un des rares clubs de Johannesburg où « les femmes non accompagnées peuvent se rendre » en dehors des réceptions privées ${ }^{68}$.

Mais les femmes queer ne sont pas les seules à considérer les domiciles privés comme des espaces sûrs. Les résidences appartenant à des Blancs sont parfois perçues comme tels par

\footnotetext{
63 Gevisser M., « A Different Fight for Freedom... », art. cité, p. 42.

64 Levi. Interview menée par Jonathan Botes, Johannesbourg, 19 août 2019, transcription en possession de l'auteur.

65 Morris A., « Race Relations and Racism... », art. cité, p. 692.

66 Gevisser M., «A Different Fight for Freedom... », art. cité, p. 19.

67 Ibid., pp. 24-25.

68 Ibid., p. 20.
} 
les hommes noirs. Les maisons deviennent des espaces qui ne sont pas seulement utilisées pour les rencontres sexuelles, mais aussi en tant que lieux de refuge contre les pass laws de I'apartheid ${ }^{69}$. Phil, un informateur de Mark Gevisser, se souvient avoir prétendu être le petit ami d'une employée de maison, ce qui, au pire, aurait valu à celle-ci d'être « accusée d'avoir enfreint le règlement sur les laissez-passer (pass laws) ; mais au moins, s'ils avaient été pris, elle [et Phil] auraient échappé à l' Immorality $A c t$, bien plus sévère ${ }^{70}$ ". Ailleurs, Peter's Place offre un lieu où de nombreux jeunes hommes trouvent refuge pour se mettre à l'abri, souvent peuplé de garçons qui ont raté le dernier train :

D'après ce que Phil raconte, vous pouviez en effet prévoir de rater le dernier train pour Soweto: "Oh mon frère, dit-il en me tapant sur la cuisse, c'était le bon temps! Sa chambre était pleine, totalement remplie de jeunes hommes craignant d'errer dans les rues la nuit ». Comme c'était souvent la coutume, Peter surélevait son lit sur des sacs de sable et des briques pour le protéger des démons, et il préparait un espace de couchage tout autour de lui pour tous ceux qui entraient. Je me souviens de la fois où la maison était si remplie que l'on ne pouvait pas ouvrir la porte. Je dormais contre la porte, mais le matin, quand je me suis réveillé, je me suis retrouvé à côté du lit, peut-être même sous le lit, parce que c'était l'époque où, si vous aviez quelqu'un de gay à côté de vous, vous rattrapiez les mois de privation durant lesquels vous n'aviez eu personne de gay à vos côtés !11

Ces espaces offrent aux jeunes homosexuels la sécurité, mais aussi des expériences sexuelles difficiles à trouver ailleurs et, à ce titre, des lieux comme Peter's Place deviennent des refuges où un garçon peut non seulement échapper aux contraintes de l'apartheid, mais aussi "trouver du réconfort auprès d'un autre garçon ${ }^{72}$ ". De toute évidence, l'accès aux maisons appartenant à des Blancs offre un certain niveau de sécurité aux citoyens noirs queer. Ces espaces sont cruciaux pour leur sécurité, eux qui sont régulièrement pris pour cible alors qu'une " contre-culture jeune, explicitement homophobe et prônant un violent "moffie-bashing"73 émerge dans les villes sud-africaines » au cours des années 1950 et se maintient tout au long de l'époque de l'apartheid ${ }^{74}$. S'il est possible de trouver des espaces sûrs grâce à d'autres personnes homosexuelles, l'accès à la ville en tant que queer peut effectivement s'avérer dangereux, les passages à tabac y étant réguliers. Exit, un magazine gay, signale une augmentation des violences homophobes à la fin des années 1980 et au début des années 199075. Levi se souvient que les lieux de drague connus dans les années 1980 ont mauvaise réputation :

Je me souviens avoir été victime d'une attaque à main armée... Je me suis senti vulnérable parce que je n'aurais pas dû être là... J'étais garé près du terrain de tennis et j'ai vu un type que j'ai trouvé plutôt mignon... et j'ai garé ma voiture [à côté de la sienne, dans des directions opposées]

\footnotetext{
${ }^{69}$ Le terme de pass laws désigne la législation sur les passeports intérieurs mise en place pour ségréguer la population, gérer le développement urbain et réguler la main-d'œuvre migrante. Elle limitait considérablement les mouvements des citoyens africains, mais aussi d'autres groupes, en leur imposant de porter un livret lorsqu'ils se trouvaient en dehors de leurs régions d'origine ou de zones particulières. Voir Shear Keith (2013), « At war with the pass laws? Reform and the Policing of White Supremacy in 1940s South Africa », The Historical Journal, 56(01), pp. 205-229.

70 Gevisser Mark (2014), Lost and Found in Johannesburg. A memoir, New York, Farrar, Straus and Giroux, p. 165.

71 Ibid., pp. 165-166.

72 lbid.

73 Terme péjoratif désignant le « gay-bashing» (dénigrement des homosexuels) en langue afrikaans.

74 du Pisani K., « Shifting Sexual Morality?... », art. cité, p. 189.

75 Batra K., « Worlding Sexualities under Apartheid... », art. cité, p. 48.
} 
... et tout à coup, il a fait marche arrière. Je ne pouvais pas savoir ce qu'il faisait, mes fenêtres étaient ouvertes et je me suis retrouvé avec un pistolet sur la tempe; un type était arrivé par derrière, [l'homme dans l'autre voiture] l'avait évidemment vu et était parti. ${ }^{76}$

Les lieux de cruising de Johannesburg sont des cibles pour le gay-bashing. Leur abandon au profit d'espaces urbains fermés a rendu les rencontres sexuelles plus sûres pour les hommes, mais les femmes ont souvent besoin de recourir à d'autres solutions pour se fréquenter, les clubs et les bars étant principalement réservés aux hommes. Les rencontres entre lesbiennes comportent aussi une autre dimension, celle de l'habillement et du style. S'Bu Kheswa, un homme trans de Soweto, qui emménage à Hillbrow dans les années 1980, révèle d'abord à sa famille son orientation sexuelle lesbienne :

[Quand j'ai fait mon coming out] il n'y pas eu de drame à ce sujet. II n'y a pas eu de remarque du type "Arrête ça », ou de risque de se faire chasser de chez soi, rien de tout ça. II n'y avait pas de telles choses. Vous savez... il n'y avait pas de problèmes parce que, traditionnellement, par exemple pour les enterrements et les trucs comme ça, les filles doivent s'habiller de telle ou telle façon, faire ceci ou cela, mais à la maison, ils ne m'ont jamais, jamais mis dans cette situation, donc je sortais en pantalon, avec mon look butch, et personne n'a jamais dit: " Tu vois bien que toutes les femmes sont couvertes, pourquoi pas toi ? ", il n'y a jamais, jamais, jamais eu de problème. ${ }^{77}$

Deux choses sont importantes à noter ici. Premièrement, grâce à la sécurité et à l'acceptation générale trouvées dans sa famille, $\mathrm{S}^{\prime} \mathrm{Bu}$ a pu faire son coming out sans être persécutée et ostracisée par les siens. Deuxièmement, $\mathrm{S}^{\prime} \mathrm{Bu}$, comme d'autres femmes queer, a fait de l'habillement un facteur déterminant de son identité et a essayé de conserver une apparence masculine :

Un jour... il y avait des gars qui se tenaient au coin de la rue, ce sont mes voisins, et alors que je passais devant eux, l'un d'eux m'a dit : " Hé, tu sais, quand je t'ai vu marcher, j'ai vraiment, vraiment cru que tu étais un gars, jusqu'à ce que tu sois plus près et que je voie ta poitrine ». Ça m'a anéanti, vous savez, ça m'a complètement anéanti, qu'il y ait quelque chose qui me trahisse [en tant que femme]. ${ }^{78}$

Divisées en deux catégories, butch et femme, les femmes queerjouent souvent des rôles normalisés au sein de leur couple, I'une accomplissant les tâches masculines stéréotypées, tandis que l'autre joue le rôle de la femme au foyer. Peut-être davantage qu'une façon pour les lesbiennes butch de signifier leur sexualité à travers leur habillement et leur apparence, la division butch/femme articule une construction sociale au sein des relations queer, mais renvoie également aux rôles qu'elles souhaitent jouer dans la société, sur leur lieu de travail et parmi les personnes extérieures à leur cercle queer.

Shay, une lesbienne blanche née dans une banlieue blanche, qui fréquente Hillbrow dans les années 1970, a vécu une expérience similaire en s'habillant de façon signifiante ${ }^{79}$ :

\footnotetext{
76 Levi. Interview menée par Jonathan Botes, Johannesbourg, 19 août 2019, transcription en possession de l'auteur.

77 S'Bu Kheswa. Interview menée Jonathan Botes, Johannesburg, 04 octobre 2019, transcription en possession de l'auteur.

78 Ibid.

79 L'utilisation de vêtements et d'autres éléments stylistiques tels que les cheveux ou le maquillage permettait aux autres de connaître sa sexualité.
} 
[Je portais] des jupes et des sortes de chemisiers et des choses comme ça. C'était inhabituel [pour moi]. Je savais que tout le monde allait me regarder parce que je n'étais pas habillée avec mon chapeau, mes gants et tout le reste. Et puis à ce moment-là, je me suis coupé les cheveux, ce qui était très inhabituel à l'époque où les femmes portaient ces franges et ces boucles sur le devant et tout ce genre de choses. Et mes parents étaient assez cool avec ça aussi... Je suis entrée dans un salon de coiffure... J'ai dit: "Solly, je veux que tu me coupes les cheveux ». II m'a regardé et m'a dit: "Ne sois pas stupide, je ne peux pas te couper les cheveux » ... J'ai contacté beaucoup de coiffeurs et ils m'ont répondu: "Non, ma chère, tes cheveux sont si beaux, ne les coupe pas, bla-bla-bla »... Et encore une fois, si vous marchiez dans la rue avec des cheveux courts, les gens vous regardaient comme si vous étiez un truc sorti d'un trou dans le sol ou quelque chose comme ça... Je travaillais dans la mécanique... [donc] c'était une excuse merveilleuse pour aller chez le médecin et dire : «Écoutez, je ne peux pas monter et descendre de ces machines en jupe, je peux porter un pantalon ? " II m'a regardée et m'a dit: «Mon Dieu, c'est une demande inhabituelle, mais si vous devez le faire, vous devez le faire. Mais faites en sorte que ce soit aussi élégant que possible. $»^{80}$

Ces anecdotes mettent en évidence la façon dont la société hétéronormative perçoit ces femmes butch, mais aussi leur volonté de conserver leur identité personnelle en dépit de réactions sociales très négatives; cette volonté est d'ailleurs confortée par des groupes de soutien que chacune d'elles mentionne. Lorsqu'elles se promènent, les femmes butch doivent être conscientes de leur environnement et des personnes avec lesquelles elles interagissent afin d'assurer leur sécurité dans une ville qui a plutôt tendance à les invisibiliser.

Faire son coming out à travers son style vestimentaire permet aux femmes de subvertir l'espace hétéronormatif qui les entoure en soulignant leur dédain manifeste pour celui-ci, et en permettant aux autres femmes d'identifier leur orientation sexuelle. Bien qu'il y ait des risques à se présenter comme butch, cela devient un moyen de montrer qui elles sont et de signifier qu'on ne doit pas les approcher; sans un tel signifiant, cela n'aurait probablement pas été possible au sein d'espaces hétéronormatifs ${ }^{81}$.

Cet article a analysé comment les personnes queer ont circulé et créé leurs propres espaces au sein d'une société qui criminalisait les sexualités non hétéronormatives. Dans un premier temps, il s'est attaché à explorer les moyens par lesquels les personnes queer ont pu définir un espace spécifique au sein de Hillbrow, et comment la vie quotidienne y a été vécue. En ayant recours à divers documents d'archives et à des entretiens réalisés avec des personnes queer qui ont vécu dans ces lieux, cette recherche a révélé que les espaces queer de Hillbrow étaient marqués par un fort dynamisme et caractérisés par de constantes transgressions. Ce qui ressort clairement de ce travail, c'est qu'il n'existe pas d'expérience homogène pour les personnes queervivant à Johannesburg au cours de la période étudiée : la classe, la race et le genre ont contribué à rendre ces expériences très diverses.

\footnotetext{
80 GALA, AM3160 (B): C4.1.1, Joburg Tracks, Tracks Interview: Shay. C'est l'auteur qui souligne.

81 S'Bu Kheswa. Interview menée par Jonathan Botes, Johannesbourg, 4 octobre 2019, transcription en possession de l'auteur.
} 
Après la promulgation de la Loi sur I'Immorality Admendment Act de 1969, les années 1970 ont vu la prolifération des clubs, dont de nombreux clubs gays à Hillbrow et alentour, à mesure que les hommes ont cherché à s'éloigner des lieux de drague de l'espace public pour trouver refuge dans la sécurité de lieux gay-friendly. Les clubs gays ont alors contribué à faire d'Hillbrow un lieu convivial pour les homosexuels de Johannesburg, mais un lieu accessible uniquement aux hommes blancs, et occasionnellement aux femmes blanches.

En raison de la stratification raciale de l'apartheid, les espaces urbains de toute l'Afrique du Sud sont alors prioritairement réservés aux Blancs, ce qui est largement le cas à Johannesburg jusqu'aux années 1980, lorsque le centre-ville commence à se déracialiser. Des endroits tels que Park Station deviennent des lieux de cruising identifiables, mais sujets à de fréquentes descentes de police; les Noirs qui s'y trouvent après la nuit tombée sont soumis aux pass laws, ce qui signifie qu'ils doivent soit quitter la ville par le dernier train, soit trouver des espaces blancs dans lesquels ils sont acceptés, comme à Peter's Place ${ }^{82}$.

Le processus progressif de déracialisation modifie l'espace queer du centre-ville. À mesure que de nombreux Blancs le quittent, les clubs queer se sont peu à peu déplacés hors du centre-ville et fait leur apparition dans les banlieues. Les personnes queer noires installées en ville trouvent refuge dans les quelques clubs qui sont restés à Hillbrow, comme le Skyline, mais doivent s'adapter à un quartier en pleine mutation.

On l'a vu, dans les années 1960, Hillbrow est un espace transgressif dominé par une population célibataire et jeune. Les cafés, les clubs et les librairies de nuit en font une destination de choix pour les banlieusards blancs qui cherchent à goûter au mode de vie bohème des habitants ${ }^{83}$. Dans cet espace de transgression, de multiples bars gays commencent à fleurir, les hommes étant contraints de se rencontrer à l'intérieur, à partir du moment où la police commence à faire des descentes régulières dans les lieux de cruising. La police ne semble pas s'opposer à la présence des clubs gays tant qu'ils restaient clos et loin des espaces visibles du public ${ }^{84}$. Cependant, les clubs et les bars ne sont accessibles qu'aux Blancs dans les années 1970. Ce n'est que dans les années 1980, avec l'effondrement du Group Areas Act à Hillbrow, que les Noirs peuvent commencer à fréquenter clubs et bars ${ }^{85}$. L'afflux des Noirs dans le quartier modifie la dynamique raciale du centre-ville, et lorsque les Blancs partent en banlieue, leurs locaux sont rapidement loués à des personnes venues de Soweto ou d'ailleurs ${ }^{86}$. Les espaces gays qui demeurent doivent modifier leur règlement pour attirer des clients, et c'est ainsi que les personnes queer noires sont autorisées à accéder à ces lieux autrefois réservés aux Blancs.

Jonathan Botes

Université du Witwatersrand (Afrique du Sud)

\footnotetext{
82 Percy. Interview menée par Jonathan Botes, Johannesbourg, 30 juillet 2019, transcription en possession de l'auteur.

83 Silverman Melinda et Zack Tanya. "Case Study: Hillbrow \& Berea », Land Use Management and Democratic Governance in the City of Johannesburg, 2007, p. 16.

84 Gevisser M., « A Different Fight for Freedom... », p. 37.

85 Dubow S., Apartheid, op. cot., p. 242.

${ }^{86}$ Murray M. J., Taming the Disorderly City..., op. cit., p. 127.
} 


\section{Bibliographie}

BANHAM Hugo (2017), « Mapping the Black Queer Geography of Johannesburg's Lesbian Women through Narrative ", PINS (Psychology in Society), 55, en ligne. URL: http://www.scielo.org.za/scielo.php?script=sci_arttext\&pid=S1015-60462017000300006 (consulté le 8 août 2021).

BATRA Kanika (2016), «Worlding Sexualities under Apartheid: From Gay Liberation to a Queer Afropolitanism », Postcolonial Studies, 19(1), pp. 37-52.

CAROLIN Andy (2017), "Apartheid's Immorality Amendment Act and the fiction of heteronormative whiteness ", Tydskrif vir Letturkunde, 54(1), en ligne. URL: http://www.scielo.org.za/scielo.php?script=sci_arttext\&pid=S0041-476X2017000100007 (consulé le 6 août 2021).

Cock Jacklyn (2003), «Engendering Gay and Lesbian Rights: The Equality Clause in the South African Constitution », Women's Studies International Forum, 26(1), pp. 35-45.

Conway Daniel (2009), «Queering Apartheid: The National Party's 1987 "Gay Rights" Election Campaign in Hillbrow », Journal of Southern African Studies, 35(4), pp. 849-863.

DuBow Saul (2014), Apartheid, 1948-1994, United Kingdom, Oxford University Press.

DU PISANI Kobus (2012), "Shifting Sexual Morality? Changing Views on Homosexuality in Afrikaner Society during the 1960s », Historia, 57(2), pp. 182-221.

GevisSER Mark (1994), "A Different Fight for Freedom: A History of South African Lesbian and Gay Organisation from the 1950s to 1990s ", in M. GEvisSER et E. CAMERON (dir.), Defiant Desire: Gay and Lesbian Lives in South Africa, Braamfontein, Ravan Press, pp. 14-88.

(2014), Lost and Found in Johannesburg. A memoir, New York, Farrar, Straus and Giroux.

Gevisser Mark et Cameron Edwin (1994), "Defiant Desire », in M. Gevisser et E. Cameron (dir.), Defiant Desire: Gay and Lesbian Lives in South Africa, Braamfontein, Ravan Press, pp. 3-13.

Hoad Neville, Karen Martin et Graeme Reid (dir.) (2005), Sex and Politics in South Africa, Cape Town, Double Storey Books.

Marcuse Peter (2016), "Whose Right(s) to what City? », in N. BRenner, P. MARCuSE et M. MAYer (dir.), Cities for People, Not for Profit: Critical Urban Theory and the Right to the City, Oxon, Routledge, pp. 2441.

MAther C. (1987), "Residential Segregation and Johannesburg's "Locations in the Sky" ", South African Geographical Journal, 69(2), pp. 119-128.

MOONEY Katie (2006), "Die Eendstert Euwel» and societal responses to white youth sub-cultural identities on the Witwatersrand, 1930-1964, Thèse de doctorat en histoire, Université du Witwatersrand.

Morrell Robert (1998), "Of Boys and Men: Masculinity and Gender in Southern African Studies », Journal of Southern African Studies, 24(4), pp. 605-630.

MORRIS Alan (1999), «Race Relations and Racism in a Racially Diverse Inner City Neighbourhood: A Case Study of Hillbrow, Johannesburg ", Journal of Southern African Studies, 25(4), pp. 667-694.

(1999), Bleakness and Light: Inner-City Transition in Hillbrow, Johannesburg, South Africa, Witwatersrand University Press. 
MURRAY Martin J. (2008), Taming the Disorderly City: The Spatial Landscape of Johannesburg after Apartheid, Ithaca and London, Cornell University Press.

Nicol Julia (1991), "Organisation of Lesbian and Gay Activists", Agenda: Empowering Women for Gender Equity, 7(11), pp. 45-46.

ReDdY Vasu (1998), « Negotiating Gay Masculinities », Agenda: Empowering Women for Gender Equity, 37, pp. 65-70.

Retief Glen (1994), "Keeping Sodom out of the Laager: The Policing of Sexual Minorities in South Africa ", in M. Gevisser et E. CAMERon (dir.), Defiant Desire: Gay and Lesbian Lives in South Africa, Braamfontein, Ravan Press, pp. 99-114.

SHEAR Keith (2013), « At war with the pass laws? Reform and the Policing of White Supremacy in 1940s South Africa », The Historical Journal, 56(1), pp. 205-229.

TABET Paola (2012), «Through the looking glass: sexual-economic exchange », in F. GRANDE OMOKARO et F. ReYsOO (dir.), Chic, chèque, choc: transactions autour des corps et stratégies amoureuses contemporaines, Genève, Graduate Institute Publications, pp. 39-51. 